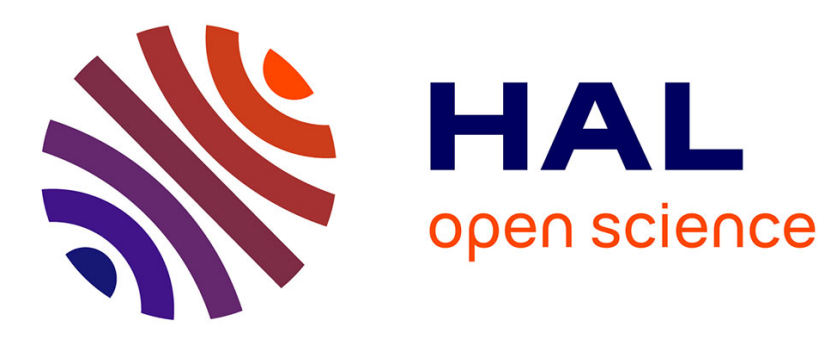

\title{
Adaptive blind estimation of sparse SIMO channels
} Abdeldjalil Aissa El Bey, Karim Abed-Meraim, Christophe Laot

\section{To cite this version:}

Abdeldjalil Aissa El Bey, Karim Abed-Meraim, Christophe Laot. Adaptive blind estimation of sparse SIMO channels. WOSSPA 2011: 7th International Workshop on Systems, Signal Processing and their Applications, May 2011, Tipaza, Algeria. hal-00609303

\section{HAL Id: hal-00609303 https://hal.science/hal-00609303}

Submitted on 18 Jul 2011

HAL is a multi-disciplinary open access archive for the deposit and dissemination of scientific research documents, whether they are published or not. The documents may come from teaching and research institutions in France or abroad, or from public or private research centers.
L'archive ouverte pluridisciplinaire HAL, est destinée au dépôt et à la diffusion de documents scientifiques de niveau recherche, publiés ou non, émanant des établissements d'enseignement et de recherche français ou étrangers, des laboratoires publics ou privés. 


\title{
ADAPTIVE BLIND ESTIMATION OF SPARSE SIMO CHANNELS
}

\author{
Abdeldjalil Aïssa-El-Bey ${ }^{1,3}$, Karim Abed-Meraim ${ }^{2}$ and Christophe Laot ${ }^{1,3}$ \\ ${ }^{1}$ Institut Télécom; Télécom Bretagne; UMR CNRS 3192 Lab-STICC, \\ Technopôle Brest Iroise CS 8381829238 Brest, France \\ ${ }^{2}$ Institut Télécom; Télécom ParisTech; UMR CNRS 5141 LTCI, \\ 37-39 rue Dareau 75014 Paris, France \\ ${ }^{3}$ Université Eeuropéenne de Bretagne, France
}

\begin{abstract}
In this paper, we focus on the adaptive identification of sparse SIMO channels in a blind context. More specifically, we propose different adaptive implementations of the sparse cross relation (SCR) method then we compare and analyse their performances in terms of convergence rate, estimation accuracy and robustness. The SCR method proceeds as follows : at first a blind approach based on the crossrelation criterion is derived for channel estimation. Secondly, to take into account the channel sparsity, the criterion is penalized by adding an extra $\ell_{p}$ norm term in order to enforce the sparsity of the desired solution. The corresponding algorithm (i.e. SCR) is shown to outperform the original CR method in terms of estimation accuracy and robustness to channel order over-estimation errors. The adaptive versions of the SCR proposed in this paper are shown to preserve the main advantages of the batch technique but suffer from low convergence rate for large dimensional systems.
\end{abstract}

Index Terms - Adaptive algorithms, stochastic gradient, sparse channel, $\ell_{p}$ norm, robustness

\section{INTRODUCTION}

Blind channel identification is a fundamental signal processing technology aimed at retrieving the channel information from its outputs only. This problem has received a lot of attention over the last two decades and many efficient solutions exist for SIMO and MIMO systems [?,?,1]. Unfortunately, these methods become inefficient (i.e. non robust or with poor estimation performance) when the channel impulse response is very long (i.e. wireless communication, echo cancelation etc), and/or sparse [2]. Such channels are encountered in many applications including underwater acoustic communication [3].

Recently some of the 'standard' blind identification methods have been modified and adapted to the sparse case either by constraining the desired solution through an $\ell_{p}$ norm based cost function with $0<p \leq 1$ which is considered as a good sparsity measure $[4,5]$ or by using a specular channel parametric model [?,2].

In this paper, we extend the work in [5] by considering the adaptive implementation of the CR and SCR and comparing the convergence speed and steady state performance of the proposed algorithms. We start by reviewing the CR and SCR methods, then we propose gradient like adaptive algorithms in a tracking scheme. In order to improve the convergence rate, we introduce an adaptive step size methodology which improves significantly the algorithm's performance at the cost of a slight increase of the computational cost.

\section{DATA MODEL}

The problem addressed in this paper is to determine the sparse impulse response of a SIMO system in a blind way, i.e. only the observed system outputs are available and used without assuming knowledge of the specific input signal.

Consider a mathematical model where the input and the output are discrete, the system is driven by a single-input sequence $s(n)$ and yields $M$ output sequences $x_{1}(n), \ldots, x_{M}(n)$, and the system has finite impulse responses (FIR's) $h_{i}(n) \neq 0$, for $n=0, \ldots, L$ and $i=1, \ldots, M$. Such a system model can be described as follows :

$$
\left\{\begin{array}{r}
x_{1}(n)=s(n) * h_{1}(n)+w_{1}(n) \\
x_{2}(n)=s(n) * h_{2}(n)+w_{2}(n) \\
\vdots \\
x_{M}(n)=s(n) * h_{M}(n)+w_{M}(n)
\end{array}\right.
$$

where $*$ denotes linear convolution and $\boldsymbol{w}(n)=$ $\left[w_{1}(n), \ldots, w_{M}(n)\right]^{T}$ is an additive spatial white noise, i.e. $\mathbb{E}\left[\boldsymbol{w}(n) \boldsymbol{w}(n)^{H}\right]=\sigma^{2} \boldsymbol{I}_{M}$ where $(\cdot)^{T}$ and $(\cdot)^{H}$ denote the transpose and the conjugate transpose, respectively and $\boldsymbol{I}_{M}$ is a $M \times M$ identity matrix. In vector form, equation (1) can be expressed as :

$$
\boldsymbol{x}(n)=\sum_{k=0}^{L} \boldsymbol{h}(k) s(n-k)+\boldsymbol{w}(n),
$$

where $\boldsymbol{h}(z)=\sum_{k=0}^{L} \boldsymbol{h}(k) z^{-k}$ is an unknown causal FIR $M \times 1$ transfer function satisfying $\boldsymbol{h}(z) \neq 0, \forall z$. Given a finite set of observation vectors $\boldsymbol{x}(1), \ldots, \boldsymbol{x}(T)$ and based on the channel entries co-primness (i.e. $\boldsymbol{h}(z) \neq 0 \forall z$ ), the objective here is to estimate the channel coefficients vector $\boldsymbol{h}=\left[\boldsymbol{h}(0)^{T}, \cdots, \boldsymbol{h}(L)^{T}\right]^{T}$ up to a scalar constant (this is an inherent indeterminacy of the blind system identification problem as shown in [1]).

\section{CR-LIKE METHODS}

\subsection{Cross-Relations method}

From equation (1), the noise-free outputs $x_{i}(n), 1 \leq i \leq M$ are given by :

$$
x_{i}(n)=s(n) * h_{i}(n), \quad 1 \leq i \leq M .
$$

Using the commutativity of convolution, it follows :

$$
h_{j}(n) * x_{i}(n)=h_{i}(n) * x_{j}(n), \quad 1 \leq i \neq j \leq M .
$$


This is a linear equation satisfied by every pairs of channels. It was shown that, based on the $\frac{M(M-1)}{2}$ possible cross-relations, the channel parameters can be uniquely identified according to [1] :

Theorem 1 Under the data model assumptions, the set of crossrelations (in the noise free case) :

$$
x_{i}(n) * h_{j}^{\prime}(n)-x_{j}(n) * h_{i}^{\prime}(n)=0, \quad 1 \leq i \neq j \leq M
$$

where $\boldsymbol{h}^{\prime}(z)$ is a $M \times 1$ polynomial vector of degree $L$, is satisfied iff $\boldsymbol{h}^{\prime}(z)=\alpha \boldsymbol{h}(z)$ for a given scalar constant $\alpha$.

By collecting all possible pairs of $M$ channels, one can easily establish a set of linear equations. In matrix form, this set of equations can be expressed as :

$$
\boldsymbol{\mathcal { X }}_{M} \boldsymbol{h}=\mathbf{0},
$$

where $\mathcal{X}_{M}$ is defined by :

$$
\boldsymbol{X}_{2}=\left[\boldsymbol{X}_{2},-\boldsymbol{X}_{1}\right]
$$

and

$$
\boldsymbol{\mathcal { X }}_{l}=\left[\begin{array}{ccc|c} 
& \boldsymbol{\mathcal { X }}_{l-1} & & \mathbf{0} \\
\hline \boldsymbol{X}_{l} & & \mathbf{0} & -\boldsymbol{X}_{1} \\
& \ddots & & \vdots \\
\mathbf{0} & & \boldsymbol{X}_{l} & -\boldsymbol{X}_{l-1}
\end{array}\right]
$$

with $l=3, \ldots, M$ and :

$$
\boldsymbol{X}_{l}=\left[\begin{array}{ccc}
x_{l}(L) & \ldots & x_{l}(0) \\
\vdots & & \vdots \\
x_{l}(T-1) & \ldots & x_{l}(T-L-1)
\end{array}\right] .
$$

In the presence of noise, equation (6) can be naturally solved in the least-squares (LS) sense according to :

$$
\widehat{\boldsymbol{h}}=\arg \min _{\|\boldsymbol{h}\|_{2}=1}\left\{\boldsymbol{h}^{H} \boldsymbol{\mathcal { X }}_{M}^{H} \boldsymbol{\mathcal { X }}_{M} \boldsymbol{h}\right\} .
$$

The CR method is referred to as the LS method in [1] because it represents the least-squares solution to the CR equation (6).

\subsection{Sparse Cross-Relations method}

In this section, we propose an iterative algorithm for the identification of sparse channels in the SIMO system case, namely the Sparse Cross-Relations method (SCR). The sparsity property can be measured by the $\ell_{p}$ norm where $0<p \leq 1$. More specifically, one can define the following sparsity based contrast function :

$$
\mathcal{J}(\boldsymbol{h})=\left\|\boldsymbol{\mathcal { X }}_{M} \boldsymbol{h}\right\|_{2}^{2}+\lambda\|\boldsymbol{h}\|_{p}^{p}
$$

where $\lambda>0$ is a weighting parameter which controls the trade-off between approximation error and sparsity. The first term is the crossrelations criterion and the second term is the penalty term which minimizes the $\ell_{p}$ norm of the channel impulse response $\boldsymbol{h}$. It is well known that the concavity of this $\ell_{p}$ norm function yields the sparse solution [6].

Therefore, the desired solution of $\boldsymbol{h}$ is determined by minimizing the cost function $\mathcal{J}(\boldsymbol{h})$ under the unit norm constraint $\|\boldsymbol{h}\|_{2}=1$ :

$$
\widehat{\boldsymbol{h}}=\arg \min _{\|\boldsymbol{h}\|_{2}=1}\left\{\boldsymbol{h}^{H} \boldsymbol{\mathcal { X }}_{M}^{H} \boldsymbol{\mathcal { X }}_{M} \boldsymbol{h}+\lambda\|\boldsymbol{h}\|_{p}^{p}\right\} .
$$

Direct minimization is computationally intensive and may be even intractable when the channel impulse response is long and when the number of channels is large. Here, a stochastic gradient technique is proposed to solve this minimization problem efficiently :

$$
\boldsymbol{h}_{k+1}=\boldsymbol{h}_{k}-\mu \nabla \mathcal{J}\left(\boldsymbol{h}_{k}\right)
$$

where $\mu$ is a small positive step size and $\nabla$ is a gradient operator. The gradient of $\mathcal{J}(\boldsymbol{h})$ is given by :

$$
\nabla \mathcal{J}(\boldsymbol{h})=\frac{\partial \mathcal{J}(\boldsymbol{h})}{\partial \boldsymbol{h}}=2 \boldsymbol{\mathcal { X }}_{M}^{H} \boldsymbol{\mathcal { X }}_{M} \boldsymbol{h}+\lambda \widetilde{\boldsymbol{h}}
$$

where

$$
\widetilde{h}(i)=p \operatorname{sign}(h(i))|h(i)|^{p-1} \quad \text { for } \quad i=1, \ldots, M(L+1) .
$$

The unit norm constraint is to ensure that the iterative algorithm do not converge to a trivial solution with all zero elements. However, we observe that the gradient of the $\ell_{p}$ norm (13) may diverge if $|h(i)|$ is close to zero and $0<p \leq 1$. Therefore, to avoid this problem, we introduce the parameter $\varepsilon>0$ in order to provide stability and to ensure that a zero-valued component in $\boldsymbol{h}_{k}$ does not strictly prohibit a nonzero estimate at the next step

$\widetilde{h}^{\varepsilon}(i)=p \operatorname{sign}(h(i))(|h(i)|+\varepsilon)^{p-1} \quad$ for $\quad i=1, \ldots, M(L+1)$,

Therefore, the update equation is given by :

$$
\boldsymbol{h}_{k+1}=\frac{\boldsymbol{h}_{k}-\mu\left(2 \mathcal{X}_{M}^{H} \boldsymbol{\mathcal { X }}_{M} \boldsymbol{h}_{k}+\lambda \widetilde{\boldsymbol{h}}_{k}^{\varepsilon}\right)}{\left\|\boldsymbol{h}_{k}-\mu\left(2 \boldsymbol{\mathcal { X }}_{M}^{H} \boldsymbol{\mathcal { X }}_{M} \boldsymbol{h}_{k}+\lambda \widetilde{\boldsymbol{h}}_{k}^{\varepsilon}\right)\right\|_{2}} .
$$

\section{ADAPTIVE IMPLEMENTATION}

For blind channel identification to be practically useful in realtime applications, it is imperative that the algorithm should be computationally simple and can be adaptively implemented. In this section, we present an adaptive implementation of the CR and penalized $\mathrm{CR}$ algorithms with least mean square (LMS) and normalized least mean square (NLMS) approache.

\subsection{Adaptive CR implementation}

In the same way that in the block approach, the cross-relations between the sensor outputs can be exploited to estimate the channel impulse responses. In this case, we can rewrite the CR criterion as follows :

$$
\overline{\mathcal{J}}(\boldsymbol{h})=\boldsymbol{h}^{H} \overline{\mathcal{Q}}_{M}(n) \boldsymbol{h},
$$

where

$$
\overline{\mathcal{Q}}_{M}(n)=\gamma \overline{\mathcal{Q}}_{M}(n-1)+\overline{\boldsymbol{X}}_{M}^{H}(n) \overline{\mathcal{X}}_{M}(n),
$$

with $\overline{\mathcal{X}}_{M}(n)$ given computed by using equations (7), (3.1) and

$$
\overline{\boldsymbol{X}}_{2}(n)=\left[\overline{\boldsymbol{x}}_{2}(n),-\overline{\boldsymbol{x}}_{1}(n)\right]
$$

and

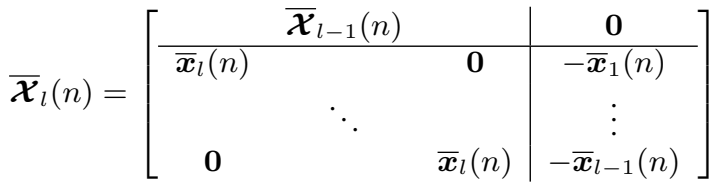

with $l=3, \ldots, M$ and :

$$
\overline{\boldsymbol{x}}_{l}(n)=\left[\begin{array}{lll}
x_{l}(n) & \ldots & x_{l}(n-L)
\end{array}\right] .
$$


Therefore, the desired solution for $\boldsymbol{h}$ is determined by minimizing the mean value of the cost function $\overline{\mathcal{J}}(\boldsymbol{h})$ :

$$
\widehat{\boldsymbol{h}}=\underset{\|\boldsymbol{h}\|_{2}=1}{\arg \min }\left\{\mathbb{E}\left[\boldsymbol{h}^{H} \overline{\mathcal{Q}}_{M}(n) \boldsymbol{h}\right]\right\}
$$

Here, an LMS algorithm is proposed to solve this minimization problem efficiently. Then, the filter coefficient vector is then update by :

$$
\boldsymbol{h}_{n+1}=\frac{\boldsymbol{h}_{n}-2 \mu \overline{\mathcal{Q}}_{M}(n) \boldsymbol{h}_{n}}{\left\|\boldsymbol{h}_{n}-2 \mu \overline{\mathcal{Q}}_{M}(n) \boldsymbol{h}_{n}\right\|_{2}} .
$$

However, the LMS algorithm suffers from slow and data-dependent convergence behavior. The normalized LMS (NLMS) [7], an equally simple, but more robust variant of the LMS algorithm, exhibits a better balance between simplicity and performance than the LMS algorithm. Therefore, we present in what follow, a NLMS approach to optimize the CR criterion. One easy way to find adaptive algorithms that adjust the new channel vector $\boldsymbol{h}_{n+1}$ from the old one $\boldsymbol{h}_{n}$ is to minimize the following function [7] :

$$
\mathcal{L}\left[\boldsymbol{h}_{n+1}\right]=d\left[\boldsymbol{h}_{n+1}, \boldsymbol{h}_{n}\right]+\mu \boldsymbol{h}_{n+1}^{H} \overline{\mathcal{Q}}_{M}(n) \boldsymbol{h}_{n+1}
$$

where $d\left[\boldsymbol{h}_{n+1}, \boldsymbol{h}_{n}\right]$ is a measure of distance from the old to the new channel vector and $\eta$ is a positive constant. The magnitude of represents the importance of correctiveness compared to the importance of conservativeness [3]. To minimize $\mathcal{L}\left[\boldsymbol{h}_{n+1}\right]$, we need to set its derivative $\frac{\partial \mathcal{L}\left[\boldsymbol{h}_{n+1}\right]}{\partial \boldsymbol{h}_{n+1}}$ to zero. Hence, the solution will be found by solving the equation :

$$
\frac{d\left[\boldsymbol{h}_{n+1}, \boldsymbol{h}_{n}\right]}{\partial \boldsymbol{h}_{n+1}}+2 \mu \overline{\mathcal{Q}}_{M}(n) \boldsymbol{h}_{n+1}=\mathbf{0}
$$

The LMS algorithm is easily obtained from (19) by using the squared Euclidean distance

$$
d\left[\boldsymbol{h}_{n+1}, \boldsymbol{h}_{n}\right]=\left\|\boldsymbol{h}_{n+1}-\boldsymbol{h}_{n}\right\|_{2}^{2}
$$

so that and from equation (19), we obtain that :

$$
\boldsymbol{h}_{n+1}=\left(\boldsymbol{I}+\mu \overline{\mathcal{Q}}_{M}(n)\right)^{-1} \boldsymbol{h}_{n},
$$

but, according to the constraint :

$$
\boldsymbol{h}_{n}^{H}\left(\boldsymbol{I}+\mu \overline{\mathcal{Q}}_{M}(n)\right)^{-1} \overline{\mathcal{Q}}_{M}(n)\left(\boldsymbol{I}+\mu \overline{\mathcal{Q}}_{M}(n)\right)^{-1} \boldsymbol{h}_{n}=0 .
$$

By using the first order approximation of the inverse matrix, the equation (22) will be :

$$
\boldsymbol{h}_{n}^{H}\left(\boldsymbol{I}-\mu \overline{\mathcal{Q}}_{M}(n)\right) \overline{\mathcal{Q}}_{M}(n)\left(\boldsymbol{I}-\mu \overline{\mathcal{Q}}_{M}(n)\right) \boldsymbol{h}_{n}=0 .
$$

Therefor, the optimal step size $\mu$ is determined as a positive solution of the second order equation :

$$
\mu^{2}-2 a \mu+b=0
$$

where

$$
a=\frac{\boldsymbol{h}_{n}^{H} \overline{\mathcal{Q}}_{M}(n)^{2} \boldsymbol{h}_{n}}{\boldsymbol{h}_{n}^{H} \overline{\mathcal{Q}}_{M}(n)^{3} \boldsymbol{h}_{n}}
$$

and

$$
b=\frac{\boldsymbol{h}_{n}^{H} \overline{\mathcal{Q}}_{M}(n) \boldsymbol{h}_{n}}{\boldsymbol{h}_{n}^{H} \overline{\mathcal{Q}}_{M}(n)^{3} \boldsymbol{h}_{n}}
$$

\subsection{Adaptive SCR implementation}

In the same way that in the adaptive standard CR implementation, the rewriting of the sparse CR criterion in adaptive case, leads to the following criterion :

$$
\overline{\mathcal{J}}(\boldsymbol{h})=\boldsymbol{h}^{H} \overline{\mathcal{Q}}_{M}(n) \boldsymbol{h}+\lambda\|\boldsymbol{h}\|_{p}^{p},
$$

Therefore, the desired solution for $\boldsymbol{h}$ is determined by minimizing the mean value of this cost function and the LMS solution is given by the following adaptive solution :

$$
\boldsymbol{h}_{n+1}=\frac{\boldsymbol{h}_{n}-\mu\left(2 \overline{\mathcal{Q}}_{M}(n) \boldsymbol{h}_{n}+\lambda \widetilde{\boldsymbol{h}}_{n}^{\varepsilon}\right)}{\left\|\boldsymbol{h}_{n}-\mu\left(2 \overline{\mathcal{Q}}_{M}(n) \boldsymbol{h}_{n}+\lambda \widetilde{\boldsymbol{h}}_{n}^{\varepsilon}\right)\right\|_{2}} .
$$

where

$\widetilde{h}_{n}^{\varepsilon}(i)=\operatorname{sign}\left(h_{n}(i)\right)\left(\left|h_{n}(i)\right|+\varepsilon\right)^{p-1} \quad$ for $\quad i=1, \ldots, M(L+1)$,

In this paper we consider only the case where $(p, \varepsilon) \in$ $\left\{(1,0),\left(0, \varepsilon_{0}\right)\right\}$ to avoid the non-convexity problem of the proposed criterion.

For the NLMS approach in the case of sparse CR criterion, we assume the same development as in section 4.1. By tackint into a count the sparsity penalty term, the new cost function can be express as :

$$
\mathcal{L}\left[\boldsymbol{h}_{n+1}\right]=d\left[\boldsymbol{h}_{n+1}, \boldsymbol{h}_{n}\right]+\mu \boldsymbol{h}_{n+1}^{H} \overline{\mathcal{Q}}_{M}(n) \boldsymbol{h}_{n+1}+\lambda\left\|\boldsymbol{h}_{n+1}\right\|_{p}^{p}
$$

In the same way that shown is section 4.1 , in order to minimize $\mathcal{L}\left[\boldsymbol{h}_{n+1}\right]$ in the case squared Euclidean distance, we need to set its derivative $\frac{\partial \mathcal{L}\left[\boldsymbol{h}_{n+1}\right]}{\partial \boldsymbol{h}_{n+1}}$ to zero. Hence, the solution will be found by solving the equation

$$
2\left(\boldsymbol{h}_{n+1}-\boldsymbol{h}_{n}\right)+2 \mu \overline{\mathcal{Q}}_{M}(n) \boldsymbol{h}_{n+1}+\lambda \widetilde{\boldsymbol{h}}_{n+1}=\mathbf{0}
$$

To avoid the divergence problem of the derivative of the $\ell_{p}$ norm, we replace the $\widetilde{\boldsymbol{h}}_{n+1}$ by $\widetilde{\boldsymbol{h}}_{n+1}^{\varepsilon}$ such as is define by equation (), and by using the following approximation $\widetilde{\boldsymbol{h}}_{n+1}^{\varepsilon} \approx \widetilde{\boldsymbol{h}}_{n}^{\varepsilon}$ for $(p, \varepsilon) \in$ $\left\{(1,0),\left(0, \varepsilon_{0}\right)\right\}$, we obtain that :

$$
\boldsymbol{h}_{n+1}=\left(\boldsymbol{I}+\mu \overline{\mathcal{Q}}_{M}(n)\right)^{-1}\left(\boldsymbol{h}_{n}-\frac{\lambda}{2} \widetilde{\boldsymbol{h}}_{n}^{\varepsilon}\right)
$$

and according to the CR constraint ;

$$
\left(\boldsymbol{h}_{n}-\frac{\lambda}{2} \widetilde{\boldsymbol{h}}_{n}^{\varepsilon}\right)^{H}\left(\boldsymbol{I}+\mu \overline{\mathcal{Q}}_{M}(n)\right)^{-1} \overline{\mathcal{Q}}_{M}(n)\left(\boldsymbol{I}+\mu \overline{\mathcal{Q}}_{M}(n)\right)^{-1}\left(\boldsymbol{h}_{n}-\frac{\lambda}{2} \widetilde{\boldsymbol{h}}_{n}^{\varepsilon}\right)=
$$

and we can find easely from the previous section that the optimal step size $\mu$ is a solution of

$$
\mu^{2}-2 \widetilde{a} \mu+\widetilde{b}=0
$$

where

$$
\widetilde{a}=\frac{\left(\boldsymbol{h}_{n}-\frac{\lambda}{2} \widetilde{\boldsymbol{h}}_{n}^{\varepsilon}\right)^{H} \overline{\mathcal{Q}}_{M}(n)^{2}\left(\boldsymbol{h}_{n}-\frac{\lambda}{2} \widetilde{\boldsymbol{h}}_{n}^{\varepsilon}\right)}{\left(\boldsymbol{h}_{n}-\frac{\lambda}{2} \widetilde{\boldsymbol{h}}_{n}^{\varepsilon}\right)^{H} \overline{\mathcal{Q}}_{M}(n)^{3}\left(\boldsymbol{h}_{n}-\frac{\lambda}{2} \widetilde{\boldsymbol{h}}_{n}^{\varepsilon}\right)}
$$

and

$$
\widetilde{b}=\frac{\left(\boldsymbol{h}_{n}-\frac{\lambda}{2} \widetilde{\boldsymbol{h}}_{n}^{\varepsilon}\right)^{H} \overline{\mathcal{Q}}_{M}(n)\left(\boldsymbol{h}_{n}-\frac{\lambda}{2} \widetilde{\boldsymbol{h}}_{n}^{\varepsilon}\right)}{\left(\boldsymbol{h}_{n}-\frac{\lambda}{2} \widetilde{\boldsymbol{h}}_{n}^{\varepsilon}\right)^{H} \overline{\mathcal{Q}}_{M}(n)^{3}\left(\boldsymbol{h}_{n}-\frac{\lambda}{2} \widetilde{\boldsymbol{h}}_{n}^{\varepsilon}\right)}
$$




\section{SIMULATIONS}

We present here some numerical simulations to assess the performance of the proposed algorithm. We consider a SIMO system with $M=3$ outputs represented by polynomial transfer function of degree $L=32$. The channel impulse response is a sparse sequence of random variables with Bernoulli-Gaussian distribution [8] :

$$
f\left(h_{i}\right)=p_{i} \delta\left(h_{i}\right)+\left(1-p_{i}\right) \frac{1}{\sqrt{2 \pi \sigma_{i}^{2}}} \exp \left(-h_{i}^{2} / 2 \sigma_{i}^{2}\right)
$$

generated by the MATLAB function SPRANDN. We used the parameters $p_{i}=0.1$ and $\sigma_{i}=1$. The input signal is a BPSK i.i.d. sequence of length $T=25000$. The observation is corrupted by addition white Gaussian noise with a variance $\sigma^{2}$ chosen such that the $S N R=\frac{\|\boldsymbol{h}\|^{2}}{\sigma^{2}}$. Statistics are evaluated over $N_{r}=100$ MonteCarlo runs and estimation performance are given by the normalized mean-square error criterion :

$$
\begin{aligned}
N M S E & =\frac{1}{N_{r}} \sum_{r=1}^{N_{r}} \min _{\alpha}\left(\frac{\left\|\alpha \widehat{\boldsymbol{h}}_{r}-\boldsymbol{h}\right\|^{2}}{\|\boldsymbol{h}\|^{2}}\right) \\
& =\frac{1}{N_{r}} \sum_{r=1}^{N_{r}} 1-\left(\frac{\widehat{\boldsymbol{h}}_{r}^{H} \boldsymbol{h}}{\left\|\widehat{\boldsymbol{h}}_{r}\right\|\|\boldsymbol{h}\|}\right)^{2},
\end{aligned}
$$

where $\widehat{\boldsymbol{h}}_{r}$ denotes the estimated channel coefficient vector at the $r^{\text {th }}$ Monte-Carlo run and $\alpha$ is a scalar factor that compensates for the scale indeterminacy of the BSI problem. For the SCR algorithm, we used SCR1 and SCR2 for the SCR algorithm with the parameters $(p, \varepsilon)=(1,0)$ and $(p, \varepsilon)=\left(0, \varepsilon_{0}\right)$ respectively.

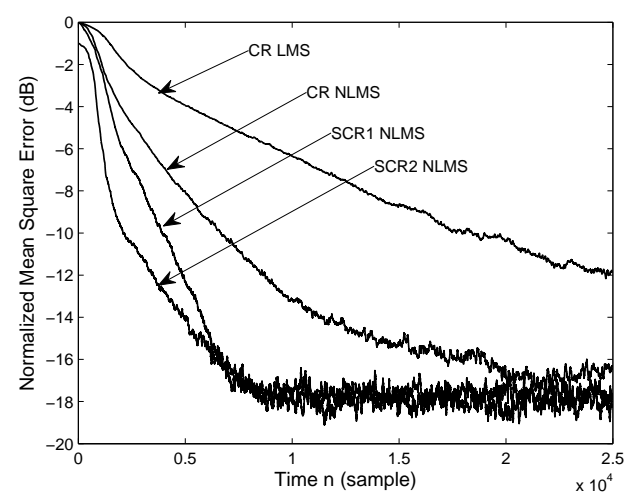

Fig. 1. Comparison of convergence between the CR and SCR adaptive algorithms for the three-channel system at $20 \mathrm{~dB}$ SNR.

\section{REFERENCES}

[1] G. Xu, H. Liu, L. Tong, and T. Kailath, "A least-squares approach to blind channel identification," IEEE Transactions on Signal Processing, vol. 43, no. 12, pp. 2982-2993, December 1995.

[2] L. Perros-Meilhac, E. Moulines, K. Abed-Meraim, P. Chevalier, and P. Duhamel, "Blind identification of multipath channels : a parametric subspace approach," IEEE Transactions on Signal Processing, vol. 49, no. 7, pp. 1468-1480, July 2001.

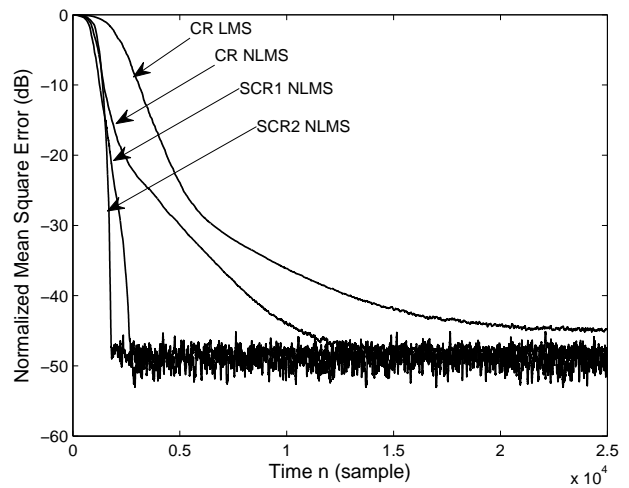

Fig. 2. Comparison of convergence between the CR and SCR adaptive algorithms for the three-channel system at $50 \mathrm{~dB}$ SNR.

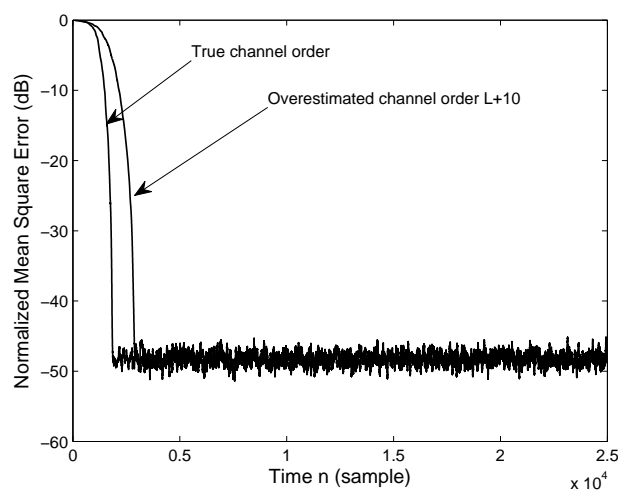

Fig. 3. Comparison of convergence of the SCR2 adaptive algorithms for the three-channel system at $50 \mathrm{~dB}$ SNR with the true channel order and overestimated channel order.

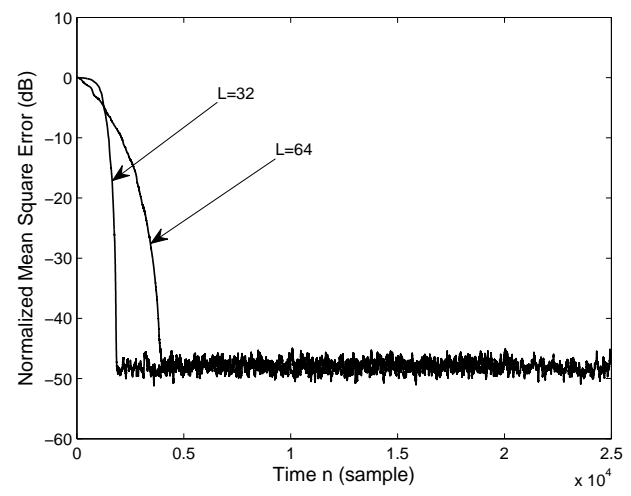

Fig. 4. Comparison of convergence of the SCR2 adaptive algorithms for the three-channel system at $50 \mathrm{~dB}$ SNR with different channel order.

[3] M. Kocic, D. Brady, and M. Stojanovic, "Sparse equalization for real-time digital underwater acoustic communications," in Proc. OCEANS, San Diego, USA, October 1995, vol. 3, pp. 14171422. 
[4] A. Aïssa-El-Bey and K. Abed-Meraim, "Blind identification of sparse SIMO channel using maximum a posteriori approach," in Proc. $16^{\text {th }}$ European Signal Processing Conference EUSIPCO, Lausanne, Switzerland, August 2008.

[5] A. Aïssa-El-Bey and K. Abed-Meraim, "Blind SIMO channel identification using sparsity criterion," in Proc. $9^{\text {th }}$ IEEE Workshop on Signal Processing Advances for Wireless Communications SPAWC, Recife, Brazil, July 2008, pp. 271-275.

[6] K. Kreutz-Delgado and B. D. Rao, "Sparse basis selection, ICA, and majorization : towards a unified perspective," in Proc. ICASSP, March 1999, vol. 2, pp. 1801-1804.

[7] S. Haykin, Ed., Adaptive filter theory, Prentice-Hall, Englewood Cliffs, NJ, 2002.

[8] M. Zibulevsky and Y. Zeevi, "Extraction of a source from multichannel data using sparse decomposition," Neurocomputing, vol. 49, no. 1, pp. 163-173, December 2002. 